\title{
Tangence
}

\section{Discours de la féminité dans The Backwoods of Canada de Catharine Parr Traill et Roughing It in the Bush de Susanna Moodie}

\section{Katherine A. Roberts}

Numéro 62, avril 2000

Parentèle

URI : https://id.erudit.org/iderudit/008172ar

DOI : https://doi.org/10.7202/008172ar

Aller au sommaire du numéro

Éditeur(s)

Presses de l'Université du Québec

ISSN

0226-9554 (imprimé)

1710-0305 (numérique)

Découvrir la revue

Citer cet article

Roberts, K. A. (2000). Discours de la féminité dans The Backwoods of Canada de Catharine Parr Traill et Roughing It in the Bush de Susanna Moodie. Tangence, (62), 34-49. https://doi.org/10.7202/008172ar d'utilisation que vous pouvez consulter en ligne. 


\section{Discours de la féminité dans The Backwoods of Canada de Catharine Parr Traill et Roughing It in the Bush de Susanna Moodie Katherine A. Roberts, Bowling Green State University}

Deux sœurs, deux visions, deux façons de vivre le Nouveau Monde: la critique a très souvent mis en parallèle les œuvres de Susanna Moodie (1803-1885) et de sa sour Catharine Parr Traill (1802-1899), non pas les premières mais certainement les deux écrivaines les plus importantes du dix-neuvième siècle au Canada. On peut même avancer que Susanna Moodie est, en fait, la véritable mère de la littérature canadienne-anglaise: cette femme compte à son actif deux volumes de nature autobiographique, plusieurs romans et textes pour enfants et une centaine de poèmes; elle est de plus collaboratrice attitrée du Literary Garland, revue mensuelle montréalaise (1838-1851). Émotive, romantique et sensible, elle s'oppose à sa sœur Catharine. Femme plus rationnelle et stoïque, celle-ci est botaniste autodidacte et également auteure de nombreux poèmes et textes de littérature enfantine. On lui doit aussi une série de six volumes d'histoire naturelle dont Canadian Wild Flowers (1868), illustré par la fille de Susanna, Agnès Fitzgibbon. Une étude des voix narratives adoptées par Catharine Parr Traill et par Susanna Moodie dans leurs textes les plus connus, The Backwoods of Canada et Roughing It in the Bush - qui traitent de la même période historique (1832-1835 et 1832-1839) — indique que les deux œuvres sont circonscrites par les discours sur la féminité qui gouvernaient le comportement des femmes de classe moyenne à l'époque prévictorienne. L'obligation pour les auteures de "naviguer" parmi ces discours crée une tension supplémentaire dans ces textes à la fois autobiographiques, didactiques et descriptifs. Les analyser en tant que récits de voyage fournit un cadre susceptible de respecter leur composition hybride et éclaire de manière nouvelle deux façons non pas contradictoires mais complémentaires de décrire le féminin au Canada.

Catharine Parr Traill et Susanna Moodie étaient respectivement cinquième et sixième filles d'une des plus remarquables familles littéraires anglaises du dix-neuvième siècle, les Strickland; 
des six filles (Agnes, Elisa, Sara, Jane Margaret, Catharine et Susanna) cinq ont, à un moment ou l'autre de leur existence, pratiqué une forme d'écriture ${ }^{1}$. Leur programme d'études comprenait non seulement les "cours" traditionnels de couture et de broderie, mais également des "cours" de lecture: la bibliothèque personnelle de Thomas Strickland leur offrait tout un éventail de titres d'histoire et d'histoire naturelle, de récits de voyages et de la littérature classique et contemporaine (anglaise, française, italienne et allemande). La disponibilité des livres et l'isolement relatif de la maison familiale, Reydon Hall, ont suscité chez les jeunes filles Strickland le goût d'écrire comme moyen de surmonter agréablement l'ennui de l'hiver. Influencées également par leurs sœurs â̂nées plus ambitieuses, Catharine et Susanna commencèrent vers 1818 à entrevoir un débouché pour leurs écrits. Catharine était en fait la première des cinq sœurs à publier un livre, The Tell Tale: An Original Collection of Moral and Amusing Stories (1818), exploit réalisé à l'âge de quinze ans. La mort du père en 1818 transforma ce qui était avant tout un passe-temps agréable en gagnepain: les sœurs tentèrent dès lors d'utiliser leur connaissance de l'histoire, de la nature et des légendes de Suffolk aussi bien que leurs propres voyages familiaux, en en faisant des livres d'instruction et de divertissement pour enfants ou des esquisses et des poèmes pour les revues littéraires élégantes qui fleurissaient en Angleterre depuis 1823.

La rencontre de Susanna et John Wedderburn Dunbar Moodie en 1830 aura pour effet de transformer la vie des deux écrivaines. Lieutenant dans les fusiliers écossais, Moodie revenait d'un séjour en Afrique du Sud avec un double but: publier un livre - Ten Years in South Africa paru en 1835 - et se trouver une femme. Susanna l'épouse en 1831 et le suit dans le HautCanada afin de prendre possession des terres que Moodie avait reçues en échange de ses services militaires. Catharine fera de même en épousant Thomas Traill, ami et camarade d'armée de

1 L'aînée, Agnes, a connu un grand succès avec The Lives of the Queens of England (12 volumes, 1840-1848), série à laquelle a également collaboré Elisa. Samuel Strickland, quant à lui, raconte ses premières années dans la forêt canadienne dans Twenty-seven Years in Canada West, or The Experience of an Early Eettler, London, R. Bentley, 1853. Je me limiterai, dans la présente étude, aux œuvres les plus connues de Catharine et de Susanna étant donné l'intérêt qu'elles offrent pour une lecture au féminin et l'influence qu'elles ont eue sur l'écriture des femmes au Canada anglais. 
36

Moodie et, comme lui, de petite noblesse écossaise. Les Traill partiront aussi à l'été 1832 pour la forêt canadienne, s'établissant près de Lakefield, Ontario, sur des terres accordées par l'armée. Une vie primitive attendait les deux sœurs. Aux difficultés inhérentes aux méthodes de culture, aux dettes constantes et à la responsabilité d'une famille en croissance rapide (Catharine a eu neuf enfants, Susanna sept) s'ajoutaient la récession de 1836-1839 et la nécessité d'assumer le fait que leurs maris respectifs - cultivés, éduqués mais plus disposés aux plaisirs de la "bonne compagnie" qu'à la construction, par exemple, d'une clôture - étaient moins bien préparés qu'elles pour les épreuves de la forêt. Même après la vente des terres et le déménagement des Traill à Peterborough en 1839 et celui des Moodie à Belleville en 1840 - où Susanna a réussi, en intervenant auprès du gouverneur Sir George Arthur, à obtenir pour son mari le poste de shérif —, la situation financière des deux familles demeurait très précaire: la publication d'une nouvelle ou d'un poème dans The Albion (New York) ou The Literary Garland (Montréal) faisait souvent la différence, du moins pour Catharine, entre avoir tout juste de quoi vivre et ne pas avoir à vendre la ferme ${ }^{2}$.

\section{Autobiographie, récit de la colonisation ou récit de voyage?}

The Backwoods of Canada (1836) et Roughing It in the Bush (1852) sont tous deux des textes d'une composition hybride. Chez Susanna Moodie, les descriptions de la traversée de l'Atlantique, du voyage jusqu'à Cobourg et de la vie en forêt sont jalonnées de poèmes et d'esquisses de personnages ou de lieux rencontrés en cours de route dont trois - "The Village Hotel", "The Land-Jobber" et "The Ould Dhragoon" — sont de la main de son mari. L'œuvre de Catharine Parr Traill, qui raconte les mêmes

2 Les détails bibliographiques sont tirés de Michel A. Peterman, "Susanna Moodie", dans Robert Lecker, David Jack et Ellen Quigley (dir.), ECW's Bibliographical Guide to Canadian Novelists, Toronto, ECW, 1993, p. 27-32; Carl Ballstadt, "Catharine Parr Traill", dans Robert Lecker et al., op cit., p. 21-26; Michel A. Peterman et Carl Ballstadt, "Introduction" à Forest and Other Gleanings. The Fugitive Writings of Catharine Parr Traill, Ottawa, University of Ottawa Press, 1994, p. 1-16, et Carl Ballstadt, Elizabeth Hopkins et Michel A. Peterman, "Introduction" à Susanna Moodie. Letters of a Lifetime, Toronto, University of Toronto Press, 1985, p. 70-73. 
événements (la traversée, le choléra à Québec, le long voyage en bateau à vapeur et en diligence), est composée de lettres envoyées à sa famille et à ses amis en Angleterre. La formulation de ces lettres permettant, chez Traill, le passage facile d'un sujet à un autre, les irrégularités de l'hiver canadien, l'absence locale d'associations poétiques, la fabrication du sucre d'érable et les habitudes de chasse des autochtones peuvent être quelques-uns des sujets traités dans une même missive. L'aspect autobiographique des écrits de Moodie et de Traill a été récemment étudié selon une approche théorique qui permet de donner une sorte d'unité à l'ensemble. La quête de soi, la construction de soi à travers l'écriture deviennent le moyen par lequel la critique cherche à faire une lecture cohérente des voix disparates qui animent ces textes ${ }^{3}$.

D'autres critiques (surtout féministes) préfèrent placer The Backwoods of Canada et Roughing It in the Bush sous la rubrique du récit de la colonisation ou settler narrative, car ils témoignent d'une manière particulièrement féminine d'affronter la terre. Pour Helen Buss, la mentalité de garnison, force qui structure l'imagination canadienne-anglaise, décrite comme l'on sait par Northrop Frye ${ }^{4}$, a été reformulée dans les travaux de John Moss $^{5}$, de Gaile McGregor et de Margot Northey au point que le

3. Voir Helen Buss, Mapping Ourselves. Canadian Women's Autobiography in English, Montréal et Kingston, McGill-Queen's Press, 1993, p. 83-104; Margaret Virginia Sampson, "Building Nation, Writing Self. The Autobiographical Writings of Anna Jameson, Susanna Moodie and Catharine Parr Traill ", mémoire de maîtrise, Dalhousie University, 1992, p. 44-89; Michael A. Peterman "Roughing It in the Bush as Autobiography", dans K. P. Stich (dir.) Reflections. Autobiography and Canadian Literature, Ottawa, University of Ottawa Press, 1988, p. 35-43.

4 Voir Northrop Frye, "Conclusion " à Carl F. Klinck (dir.), Histoire littéraire $d u$ Canada, Québec, Presses de l'Université Laval, 1970, p. 982: "De petites communautés isolées et entourées d'une "frontière" physique ou psychologique, séparées les unes des autres et séparées aussi de leurs sources culturelles américaine et britannique: des communautés qui fournissent tout ce que leurs membres possèdent en fait de valeurs foncièrement humaines et qui se voient forcées d'éprouver un grand sentiment de respect pour la loi et l'ordre qui les tiennent unies ensemble, et qui, incapables de penser, se trouvent cependant aux prises avec un cadre physique immense, menaçant et redoutable - de telles communautés sont destinées à développer ce que nous pouvons appeler provisoirement une mentalité de garnison."

5 Patterns of Isolation in English Canadian Fiction, Toronto, McClelland and Stewart, 1974, p. 16-34. 
paysage canadien est souvent associé au côté sombre de l'âme ${ }^{6}$. Selon McGregor, l'horreur de la terre était à ce point grande que la nature est devenue démythifiée, invisible, neutralisée ${ }^{7}$. En vingt ans, cette réaction limitée des Canadiens anglais face à la nature s'est transformée, selon Buss, en une vertu: leur capacité d'effacer l'environnement dans l'imaginaire et de le nommer, le rebaptiser à l'image de l'homme, en fait foi ${ }^{8}$. Or, les narratrices de plusieurs textes autobiographiques de femmes - dont ceux de Traill et de Moodie — réagissent à l'étrangeté du paysage canadien en essayant de s'identifier à la terre. L'expérience féminine du Canada indique que le fait d'être entouré par la forêt est une expérience positive qui exige un recours à des métaphores de nature plus érotique et maternelle que celles employées par des hommes?.

Autobiographie ou récit de la colonisation? La critique continuera sans doute à débattre de l'appartenance générique de ces textes. Les aborder uniquement par le biais de l'autobiographie sert à obscurcir les visées publiques de chaque ouvre, dans la mesure où celles-ci agissaient en tant que guide pratique destiné aux émigrants anglais de bonne société qui désiraient s'établir au Canada. Le fait qu'au dix-neuvième siècle des colons d'origine britannique viennent s'établir en Amérique du Nord ne constituait pas une rupture des liens avec ce "vieux pays", mais un déplacement du centre vers la périphérie et apparaissait ainsi le besoin concomitant d'expliquer la vie de la périphérie à ceux du centre ${ }^{10}$. C'est dans ce sens qu'on peut envisager The Backwoods of Canada et Roughing It in the Bush comme des récits de voyage qui offrent une version légère mais informative des mours et

6 Margot Northey, The Haunted Wilderness. The Gothic and the Grotesque in Canadian Fiction, Toronto, University of Toronto Press, 1976, p. 8.

7 The Wacousta Syndrome. Explorations in the Canadian Landscape, Toronto, University of Toronto Press, 1985, p. 71-72.

8 Helen M. Buss, "Women and the Garrison Mentality. Pioneer Women Autobiography and their Relation to the Land", dans Lorraine McMullen (dir.), Re(dis)covering our Foremothers. Nineteenth-century Canadian Women Writers, Ottawa, University of Ottawa Press, 1990, p. 124. Je traduis.

9 Ibid., p. 133. Voir aussi Elizabeth Thompson, The Pioneer Woman. A Canadian Character Type, Montréal et Kingston, McGill-Queen's Press, 1991, p. 30-59.

10 D. M. R. Bentley, "Breaking the Cake of Custom. The Atlantic Crossing as a Rubicon for the Female Emigrants to Canada?", Lorraine McMullen, op. cit., p. 119. Je traduis. 
coutumes des habitants du nouveau continent, y compris ceux des autochtones ${ }^{11}$.

Le récit de voyage, ancien ou contemporain, a connu un regain d'intérêt considérable au cours des dernières années surtout dans le domaine des études postcoloniales. Contrairement à la croyance populaire qui stipulait que la femme victorienne échappait rarement à la sphère domestique, un grand nombre de femmes ont voyagé et ont rédigé des récits de voyage. Cet aveuglement face à cette abondante production féminine tient, selon Sara Mills, au fait que

dans le contexte colonial, les femmes britanniques n'étaient pas autorisées à jouer un rôle autre que celui de la figuration symbolique du foyer et de la pureté; la notion de la femme comme participante active était à peine imaginable ${ }^{12}$.

Dans Discourses of Difference. An Analysis of Women's Travel Writing and Colonialism, Mills se livre à une analyse fouillée du contexte de production et de réception des récits de voyage rédigés par des femmes à l'époque impérialiste. En dépit de leur grande hétérogénéité, la critique (surtout masculine) a tendance à en présenter une vision homogène:

Les récits de voyage de femmes constituent une transcription fidèle de leurs aventures, les auteures elles-mêmes, dont la critique fait le portrait d'après le contenu du récit, sont qualifiées d'indomptables, d'excentriques et de folles ${ }^{13}$.

Mais, par contre, quand il est question de voyages exigeants et de situations dangereuses, leur version des événements est souvent jugée fausse comme si de tels exploits dépassaient la force physique d'une femme. En somme, le récit de voyage des femmes britanniques du dix-neuvième siècle est au carrefour de deux discours hétérogènes: le discours féminin et le discours colonial. Leurs textes sont donc le produit d'une configuration complexe

11 Le peu d'espace dont je dispose ici ne me permet pas d'analyser en détail le portrait que dessinent Moodie et Traill des Chippewas du lac de Riz. Pour une analyse de cet aspect de leurs ouvres, voir l'excellent article de Carole Gerson, "Nobler Savages. Representations of Native Women in the Writings of Susanna Moodie and Catharine Parr Traill", Journal of Canadian Studies, Peterborough (Ontario), vol. 32, nº 2, été 1997, p. 5-21.

12 Sara Mills, Discourses of Difference. An Analysis of Women's Rravel Writing and Colonialism, London, Routledge, 1991, p. 3. Je traduis.

13 Ibid., p. 32. Je traduis. 
40

de structures discursives parmi lesquelles l'auteure doit manouvrer. Le discours de la féminité, qui prônait, entre autres, la modestie, la chasteté, la maîtrise rigide des passions et la frugalité en matière d'économie domestique, s'est révélé particulièrement tenace et problématique pour ces deux premières sours de la littérature canadienne-anglaise.

\section{The Backwoods of Canada: l'échec de l'ange du foyer?}

Le plus important texte de Catharine Parr Traill, The Backwoods of Canada. Being Letters from the Wife of an Emigrant Officier, Illustrative of the Domestic Economy of British America, a été publié en Angleterre en 1836 et traduit en français sous le titre: Les forêts intérieures du Canada, lettres écrites par la femme d'un officier émigrant sur la vie domestique des colons Américains en $1843^{14}$. La structure épistolaire du récit lui confère un ton spontané. Or, en dépit de l'aspect authentique ou innocent d'une première impression qui n'est pas teintée d'une sagesse rétrospective, il faut rappeler que le récit de voyage par lettres est une convention littéraire. Étant donné ce qu'on sait des finances précaires de la famille Traill, il est tout à fait probable que Catharine ait songé dès le début à publier ses lettres, plusieurs d'entre elles étant scrupuleusement composées en vue de cette publication. "Optimisme", "rationalité", "esprit pratique" sont les mots d'ordre de la narratrice qui ne voudrait à aucun prix avouer la faiblesse, la dépression ou le mal du pays que son exil canadien a dû provoquer par moments. Certains sujets d'écriture étaient évidemment tabous: les femmes n'étaient pas censées connaître la sexualité, et en parler dans leurs textes était impensable. Catharine Parr Traill passe ainsi sous silence presque tout ce qui touche à sa vie familiale et à son rapport avec son mari ${ }^{15}$.

Se conformant aux stéréotypes inhérents aux sujets féminins, la voyageuse du dix-neuvième siècle passait souvent son temps à décrire, par exemple, des papillons et des fleurs tropicales. Selon Joanna Trollope, les plantes, en autant qu'elles ne soient pas trop sauvages et donc trop sexuelles, étaient un sujet acceptable, pro-

14 Paris, L. Curmer éditeur, 1843. Toutes les références subséquentes à ce texte sont tirées de cette édition.

15 Voir Michel A. Peterman et Carl Ballstadt, "Introduction" à Forest and Other Gleanings, loc. cit., p. 6-9. 
prement asexué et juste assez esthétique, sans être trop intellectuel ${ }^{16}$. La narratrice de The Backwoods of Canada nourrissant un vif intérêt pour les fleurs sauvages de son pays d'adoption, plusieurs lettres du volume sont remplies de descriptions de ses randonnées dans la forêt et des spécimens nouveaux qu'elle y trouvait. Or, son intérêt pour le monde naturel dépasse de loin celui d'un passe-temps anodin comme en témoigne la sixième lettre envoyée à sa mère, lettre dans laquelle elle propose de former pour sa sœur Elisa un herbier (bortus siccus) assorti d'une description détaillée des plantes, de leur taille et de leurs qualités:

Je ferai des notes sur toutes les particularités frappantes qui les concernent; dites-lui qu'elle peut compter que je lui enverrai, par la première occasion, les échantillons et les graines que je pourrai recueillir (p. 36).

Comme l'a montré Sara Mills, les récits de voyage des femmes de cette époque ne sont pas censés être de nature "scientifique" ni autoritaire, mais plutôt de nature amateur ${ }^{17}$. La contradiction entre le contenu sérieux et scientifique du texte et les contraintes imposées par le discours de la féminité se résout très souvent ici dans un discours d' "auto-dénigrement", présent aussi chez sa sœur Susanna, où l'auteure minimise l'importance de ses découvertes. Catharine écrit ceci à sa mère: "Je crois que nos savants botanistes anglais me trouveraient bien présomptueuse de donner ainsi des noms aux fleurs et aux plantes que je rencontre dans ces forêts sauvages" (p. 40). Et encore à sa sœur :

[...] je me lamente chaque jour de ne pouvoir représenter fidèlement les fleurs de ma patrie adoptive, ou de ne pas connaitre la classification botanique comme vous pourriez le faire. J'ai appris à en connaître quelques-unes, mais j'ose à peine me fier à mon faible savoir et me hasarder dans des descriptions scientifiques, lorsque je sens qu'une erreur serait aisément découverte, et m'exposerait au ridicule et au mépris (p. 94).

Cette incursion féminine dans le domaine de la nomenclature à un moment dans l'histoire où existait une séparation nette entre les sphères d'influence masculine (publique) et féminine (domestique) est importante. Traill montre ainsi une volonté de comprendre son espace physique et de s'en accommoder; l'intérêt

16 Joanna Trollope, Britannia's Daughters. Women of the British Empire, cité dans Sara Mills, op. cit., p. 81. Je traduis.

17 Sara Mills, ibid., p. 83. 
pour les graines, mentionné ci-haut, traduit un désir d'adaptation, un désir de prendre racine, comme les plantes qu'elle collectionne. Traill n'est toutefois pas sans reconnaitre l'audace d'une telle entreprise de savoir féminin. Les limites qu'elle impose à sa propre typologie naturelle en disent long sur les jugements qui pèsent sur la valeur scientifique des écrits de femmes.

D'autres tensions se font sentir dans les passages consacrés à la différence sociale en sol canadien et dans ceux qui sont destinés à avertir ses concitoyens britanniques des privations de la vie dans les forêts intérieures. Il faut comprendre que Traill comme Moodie étaient des femmes de bonne société, toutes les deux épouses d'un officier de petite noblesse et habituées à un traitement de faveur tel qu'il seyait aux femmes de leur rang. La narratrice de The Backwoods of Canada a vite compris que la situation particulière de la colonie canadienne-anglaise nécessitait une redéfinition de la hiérarchie sociale dans ce pays où les teneurs de magasins appartenaient à des familles fort distinguées :

comme ils tiennent dans la société un rang qui les met sur le pied d'égalité avec l'aristocratie du pays, vous ne serez pas surprise quand je vous dirai qu'il n'est pas extraordinaire de voir des fils d'officiers et des dignitaires ecclésiastiques derrière un comptoir, ou maniant la hache dans les forêts avec les bûcherons de leur père; et ces occupations ne leur enlèvent point leur rang dans la société. Après tout c'est l'éducation, ce sont les manières qui doivent distinguer l'homme comme il faut [...] L'ignorant, quelque riche qu'il soit, ne peut jamais être l'égal de l'homme bien élevé. C'est l'esprit qui fait, dans ce pays, la distinction des classes. "Le pouvoir est dans le savoir" (p. 33).

La nouveauté du pays et le besoin des gens honnêtes et industrieux obligeaient les gens des classes aisées à remplir de nouveaux offices, ce qui aurait paru incongru en Angleterre. Or, autant Traill accepte ce nouveau système qui est très avantageux pour le développement de la colonie, autant elle se montre réticente à renoncer à l'idée de supériorité des gens de son rang dont la pierre angulaire est l'éducation. Catharine Parr Traill et Susanna Moodie sont toutes les deux pour un système "méritocratique" où l'individu sensé et éduqué pourrait exercer une influence sur les politiques gouvernementales en raison de sa vertu individuelle. Mais la narratrice est beaucoup plus exigeante envers les femmes qu'envers les hommes qui décident de tenter l'aventure de l'émigration; elle ira même jusqu'à blâmer les fem- 
mes pour l'échec des tentatives de colonisation. Si les défricheurs masculins s'accommodent petit à petit de leur nouvelle situation, ils

se plaignent que leurs femmes regrettent toujours la patrie, et se lamentent d'avoir traversé les mers. [...] Fort peu entrent de bon cour dans la vie des nouveaux colons. [Les femmes] manquent de petites douceurs domestiques auxquelles elles avaient été accoutumées (p. 41).

L'importance pour Traill de la participation féminine dans l'aventure de la colonisation tient de la conception de la femme comme "ange du foyer" telle qu'elle apparait dans les discours sur la féminité au dix-neuvième siècle. La récente étude de Misao Dean, Practicising Femininity. Domestic Realism and the Performance of Gender in Early Canadian Fiction ${ }^{18}$, aide à comprendre l'aspect contradictoire du texte de Traill qui semble lutter simultanément pour et contre la reconnaissance de la contribution des femmes à la construction du Nouveau Monde. L'écriture de Traill dans The Backwoods of Canada et dans The Female Emigrant's Guide, and Hints of Canadian Housekeeping (1854) constitue une sorte de "panier de reprisage" à partir duquel l'auteure tâche de refaire les liens entre le comportement économique exigé des nouvelles arrivantes et la notion d'une essence féminine immuable. Caractérisée par la maîtrise de soi et le contrôle du désir, la femme au foyer contrôlait les dépenses de la maison en maîtrisant ses propres désirs de consommatrice; ces qualités, selon l'idéologie, permettaient aux femmes de régler le comportement indiscipliné des hommes. Le statut moral naturellement supérieur des femmes les autorisait à réprimer, à surveiller le comportement masculin à la fois à l'intérieur et à l'extérieur du foyer ${ }^{19}$. Il s'agissait pour ces femmes "féminines", dépositaires des vertus de la patience chrétienne, de veiller à toutes les tâches domestiques et à la sauvegarde des valeurs morales et spirituelles de la famille. Bref, elles étaient responsables du bonheur du mari et du succès de l'entreprise coloniale. Si l'éducation est une vertu chez l'homme de son rang, cette même qualité, si chère à Traill comme ultime mesure des différences sociales, sera la ruine de la femme. C'est justement la femme instruite, cultivée, bourgeoise, raffinée qui vit le plus difficilement l'adaptation à la vie dans la forêt:

18 Toronto, University of Toronto Press, 1998.

19 Ibid., p. 21. 
Elle soupire après ces petits agréments domestiques, cet extérieur de raffinement et de luxe qu'elle avait coutume de voir autour d'elle. Il lui reste alors peu de temps pour ces occupations qui étaient son unique affaire aussi bien que son plaisir. Les perfections qu'elle doit acquérir sont d'un ordre différent. Il faut qu'elle devienne habile dans l'art de faire le sucre, la chandelle et le savon; qu'elle apprenne à cuire d'énormes pains, etc. (p. 75).

Le fait d'éreinter les femmes - et surtout celles de sa propre classe - pourrait être vu comme une façon, de la part de Traill, de laisser libre cours à ses propres frustrations et de parler indirectement de son propre malheur. Après tout, sa situation dans la forêt était loin d'être romantique: la maladie, l'isolement, de nombreuses grossesses et un mari enclin à des périodes de dépression sérieuse ont dû mettre ses bonnes dispositions à rude épreuve $^{20}$. La rigueur de la vie dans la forêt nécessitait une adaptation radicale qu'une femme éduquée - comme Traill ellemême - était mal préparée à réussir. Les codes de bienséance qui dictaient le type de conduite acceptable pour une femme issue de la classe moyenne l'empêchaient, plus que son homologue masculin, d'exécuter certaines tâches essentielles à la survie de la famille. Cet aspect des discours sur la féminité, assimilé par Traill, fait donc obstacle au genre d'adaptation qu'elle préconise.

\section{Roughing It in the Bush: pour un Robinson Crusoé au féminin}

S'il est vrai que la narratrice de The Backwoods of Canada ne s'appesantit pas sur les malheurs familiaux, la voix narrative qu'adopte sa sour Susanna fait des souffrances des Moodie le centre de son récit. Chez elle, aucun effort n'est fait pour cacher ses émotions, ses peurs, son mal du pays. Roughing It in the Bush se compose de trente chapitres qui traitent de la traversée, de l'arrivée à Québec, du voyage jusqu'au Haut-Canada et des années de défrichement dans la forêt près de Peterborough. Racontant ses aventures une dizaine d'années plus tard, sous la forme d'un journal, Susanna a choisi de souligner à la fois le manque de confort et la richesse psychologique de son expérience canadienne.

20 Or ces aspects de sa vie ne sont pas décrits dans The Backwoods... Voir Michel A. Peterman et Carl Ballstadt, loc. cit., p. 5-7. 
Après avoir vécu deux ans sur une ferme près de Cobourg, les Moodie ont déménagé à Lakefield afin d'être près des familles Traill et Strickland. Susanna a recueilli de la documentation sur sa première marche en forêt, en compagnie de sa sœur toujours "joyeuse et enthousiaste" qui l'encourage à voir d'un "œeil favorable" les terres marécageuses sur lesquelles sera construite leur hutte primitive ${ }^{21}$. Pour Susanna, le fait d'évoquer sa sœur courageuse sert à mettre en relief sa propre timidité, sa propre peur face à l'idée d'une vie dans la forêt.

Roughing It in the Bush est, comme The Backwoods of Canada, un texte au carrefour des genres romanesque, intime et documentaire. Si Moodie cherche d'une part à reproduire assez fidèlement les circonstances de son installation au Canada dans le but de décourager d'autres émigrants de sa classe à investir leur argent dans des terres non défrichées, elle cherche d'autre part à donner une unité fictive à cet ensemble de chapitres et à créer un récit d'aventures à la Robinson Crusoé dans lequel elle joue le rôle de l'hérö̈ne. La référence implicite, dans le chapitre "Tom Wilson's Emigration", au roman célèbre de Daniel Defoe, donc au récit de voyage, permet d'élargir la classification générique de Roughing It in the Bush, texte le plus souvent étudié en tant qu'autobiographie. Cette façon de faire d'une femme qui s'embourbe dans ses prétentions littéraires britanniques, tiraillée par une forêt qui la terrifie et la fascine, a abouti non pas à une unification des "voix narratives" parfois contradictoires du texte, mais à leur polarisation. Mettre le texte dans le cadre du récit de voyage de femmes éclaircit plusieurs de ces ambiguités narratives, surtout celles qui tournent autour de la figure héroïque dans laquelle Susanna essaie de se projeter. Ces ambiguités ou contradictions seraient le résultat d'un conflit entre la conception intériorisée de l'idéal féminin et l'énergie active de la narratrice à un moment dans l'histoire où les femmes n'étaient pas censées montrer la moindre assurance. Les discours sur la féminité visaient à circonscrire les mouvements de la femme de la classe moyenne - une femme respectable, par exemple, ne sortant jamais sans chaperonne - afin de la protéger des désirs masculins imprévisibles. En

21 Susanna Moodie, Roughing it in the Bush, Toronto, McClelland \& Stewart, "New Canadian Library", 1991, p. 277. Toutes les références entre parenthèses sont tirées de cette édition et traduites par moi. Catharine et Susanna étaient des voisines de 1834 à 1839. Si Susanna en parle souvent, Catharine n'en parle qu'une fois (p. 129). 
46

somme, ces femmes étaient vues comme faibles et souffrant de toutes sortes de maladies débilitantes, incapables de se débrouiller toutes seules et dépendantes de la protection masculine ${ }^{22}$.

La voix narrative de Susanna Moodie montre que l'auteure a en partie assimilé cette notion d'impuissance féminine: elle évoque souvent sa faiblesse et dessine d'elle-même le portrait d'une femme peureuse et pleurnicharde. Or, un écart se fait jour dans le texte entre ce discours de la féminité et les nombreuses actions hérö̈ques de Moodie : un écart entre Susanna la narratrice et Susanna le personnage. Tout comme sa sœur se complaisait à minimiser ses connaissances en histoire naturelle, Moodie cherche à réduire la portée de ces exploits. En fait, elle fait preuve de beaucoup de courage face au danger. Au début du récit, dans le chapitre intitulé "Québec" où elle relate la collision entre le Anne, bateau sur lequel les Moodie avaient traversé l'Atlantique, et un autre navire, Susanna ordonne aux passagères — folles de peur — de se calmer et de se réfugier en bas pendant que les marins font les réparations nécessaires (p. 44-45). Elle se plaît également à raconter comment elle a appris à manier un canot, bien que "[s]a connaissance de l'usage de l'aviron n'était pas entièrement sans danger" (p. 470), et à transporter des passagers de l'autre côté du lac de Riz, même au cour de l'orage. Elle rentre saine et sauve, dirigeant seule sa barque et remontant un fort courant malgré ce qu'elle appelle "sa main faible de femme" (p. 471). Que ce soit dans sa description de l'incendie de la jachère ou de l'incendie menaçant la maison familiale, Moodie se plaît à créer de chaque événement un épisode dramatique avec héros, scélérats et intervention divine. Or, c'est Susanna elle-même qui est au centre du récit, la mère responsable du bien-être de la famille et de la protection des enfants qui garde son sang-froid au milieu du "vacarme" (p. 308). Voici la description qu'elle offre du deuxième incendie:

Les nouvelles de l'incendie se sont propagées de tous côtés. On disait que j'avais fait des miracles et que j'avais mis à l'abri la plupart des meubles avant l'arrivée des secours. Réduites à un récit sans ambages, ces merveilles se rapetissent au simple et non pas merveilleux fait que, pendant les événements, j'ai déplacé des coffres, que, en temps normal, je n'aurais pas pu transporter; et que j'étais inconsciente à la fois du froid et du danger auxquels j'étais exposée, travaillant sous un toit en flam-

22 Sara Mills, op. cit., p. 95. Je traduis. 
mes qui, lui, s'il était tombé, nous aurait enterrés moi et mes enfants (p. 394).

En minimisant l'importance de ses actions dans le contexte d'un événement dont elle est le personnage central, Moodie attire l'attention du lecteur sur ce qu'elle fait. Et elle se débrouille en forêt bien mieux qu'il n'y paraît à première vue. La tendance à réduire la portée de ces exploits se traduit par un discours d'autodénigrement - tout comme dans les propos de sa sour Catharine - , auto-dénigrement qui serait le résultat de l'impossibilité de conjuguer les discours sur la féminité et la narration d'aventures masculines sur laquelle est basé le récit de voyage.

\section{Catharine, Susanna et leur descendance littéraire féminine}

Femmes héroïques ou passives, féministes ou traditionnelles, Catharine Parr Traill et Susanna Moodie ont légué à la littérature canadienne-anglaise des portraits chargés de contradictions qui continuent de fasciner à la fois la critique et les créateurs littéraires. C'est peut-être à cause de leur statut énigmatique de femmes seules dans la forêt canadienne qu'elles ont inspiré tant d'écrivaines. Elles fournissent à leurs "filles" littéraires un modèle de comportement, une façon d'être canadienne à laquelle des auteures plus contemporaines peuvent se mesurer. Margaret Atwood, une des plus grandes auteures canadiennes-anglaises, remarque, en parlant ici de toutes les femmes du pays: "On aimerait croire qu'on aurait ressemblé, dans les mêmes circonstances, à Catharine Parr Traill avec son esprit pratique imperturbable, [mais] la plupart d'entre nous soupçonnons, de manière subreptice, qu'on aurait ressemblé plus à Susanna Moodie ${ }^{23}$." Atwood semble être fascinée en fait par la séparation - la "schizophrénie" — des voix narratives dans Roughing It in the Bush. Selon elle, l'image de Moodie qui se dégage de ce texte est celle d'une "dualité violente ${ }^{24}$ ", d'un archétype de la condition canadienne-anglaise qui

23 Introduction à Roughing it in the Bush, cité dans D.M.R. Bentley, loc. cit., p. 114. Je traduis.

24 Margaret Atwood, The Journals of Susanna Moodie, Toronto, Oxford University Press, 1970, p. 64. Voir aussi Eva-Marie Kröller, "Resurrections: Susanna Moodie, Catharine Parr Traill and Emily Carr in Contemporary Canadian Literature", Journal of Popular Culture, Bowling Green (Ohio), vol. 15, $\mathrm{n}^{\circ} 3$, 1981, p. 39-42. 
refuse et accepte simultanément la présence de la nature. Chez Atwood, le personnage de Susanna ressent une difficulté à garder une prise sur le réel et exprime la perte de l'identité et l'isolement. Moitié hommage, moitié satire d'une mère littéraire, les poèmes d'Atwood, en dépit de leur iconographie brillante et de leur puissance littéraire saisissante, reposent sur la conception populaire qui fait de Susanna Moodie une névrosée ${ }^{25}$. Les écrits de Moodie continuent toutefois de marquer l'écriture de cette grande dame des lettres canadiennes contemporaines. Atwood affirme avoir trouvé l'inspiration pour son roman historique Alias Grace (1996), roman qui traite du procès de la jeune servante Grace Marks pour le meurtre de son employeur, dans la version que raconte Susanna Moodie de ce fait divers dans la suite à Roughing It in the Bush. Life in the Clearings Versus the Bush (1853) ${ }^{26}$.

Les écrits de Catharine Parr Traill ont donné naissance, si je puis dire, à moins de "filles littéraires" que ceux de sa sour Susanna, sans doute à cause de leur nature plus documentaire. La "voix" de Catharine se fait entendre toutefois dans le roman The Diviners (1974) de Margaret Laurence, autre grande dame des lettres canadiennes, mais de manière différente. C'est au tempérament, non pas divisé et indécis de Susanna, mais rationnel et courageux de Catharine que fait appel la narratrice Morag Gunn, elle-même auteure, quand elle invoque "Sainte Catharine". Le langage suranné et cérémonieux de Catharine dans Backwoods, évoqué dans le roman de Laurence, se heurte à l'argot coloré et blasphémateur de Morag qui évoque son ancêtre à la fois comme modèle à suivre et comme héritage à dépasser dans la quête de sa propre voix littéraire ${ }^{27}$.

L'interprétation largement autobiographique de The Backwoods of Canada et de Roughing It in the Bush a contribué à mettre en place une vision de ces textes comme portrait fidèle de leurs auteures: de là, il est tentant d'en arriver à la conclusion que Catharine était mieux adaptée aux privations de la vie dans la forêt que

25 Fiona Sparrow, "This Place is Some Kind of Garden". Clearings in the Bush in the Works of Susanna Moodie, Catharine Parr Traill, Margaret Atwood and Margaret Laurence", The Journal of Commonwealth Literature, Londres, vol. $25, \mathrm{n}^{\circ} 1,1990$, p. 27.

26 Voir Margaret Atwood, In Search of Alias Grace. On Writing Canadian Historical Fiction, Ottawa, Presses de l'Université d'Ottawa, 1997, p. 28-34.

27 Margaret Laurence, The Diviners, Toronto, McClelland \& Stewart, "New Canadian Library", 1974, p. 430-431. 
sa sœur Susanna. Or, de telles lectures laissent de côté les contraintes imposées à leurs écrits par les discours en circulation à l'époque prévictorienne, notamment ceux qui dictaient les codes d'un comportement acceptable aux femmes de la classe moyenne. Catharine Parr Traill, tout en s'aventurant, à travers ses découvertes, dans le domaine des sciences naturelles, s'est trouvée aux prises avec une conception de la féminité qui l'empêchait de participer, de tout cour, à l'entreprise de colonisation. Susanna Moodie, quant à elle, tout en minimisant sa participation aux aventures de la forêt, parvient à insérer son rôle d'épouse et de mère dans un cadre héroïque.

Ces deux premières sœurs de la littérature canadienne ont livré, en somme, dans la forme de The Backwoods of Canada et de Roughing It in the Bush, deux textes fracturés, contradictoires même. C'est justement cet héritage de contradiction, propre à la condition féminine du dix-neuvième siècle, qui agit en tant que fonds culturel et littéraire dans lequel de nombreuses écrivaines canadiennes continuent à puiser. 